\title{
Autophagy Controls Acquisition of Aging Features in Macrophages
}

\author{
Amanda J. Stranks ${ }^{\text {a }}$ Anne Louise Hansen ${ }^{b}$ Isabel Panse ${ }^{a}$ \\ Monika Mortensen ${ }^{e}$ David J.P. Ferguson ${ }^{c}$ Daniel J. Puleston ${ }^{a}$ \\ Kevin Shenderov ${ }^{f}$ Alexander Scarth Watson ${ }^{a}$ Marc Veldhoen $^{d}$ \\ Kanchan Phadwal $^{b}$ Vincenzo Cerundolo ${ }^{a}$ Anna Katharina Simon ${ }^{a}$ b \\ ${ }^{a}$ MRC Human Immunology Unit, Weatherall Institute of Molecular Medicine, University of Oxford, ${ }^{\mathrm{b}} \mathrm{BRC}$ Translational \\ Immunology Lab, Experimental Medicine, Nuffield Department of Medicine, and ' Nuffield Department of Clinical

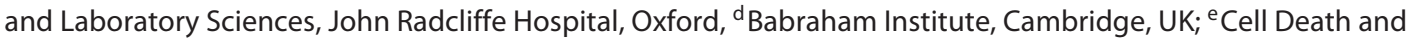 \\ Metabolism, Danish Cancer Society Research Center, Danish Cancer Society, Copenhagen, Denmark; ${ }^{f}$ Johns Hopkins \\ University School of Medicine, Baltimore, Md., USA
}

\section{Key Words}

Autophagy · Macrophages · Aging · Inflamm-aging · Inflammation $\cdot$ Metabolism

\begin{abstract}
Macrophages provide a bridge linking innate and adaptive immunity. An increased frequency of macrophages and other myeloid cells paired with excessive cytokine production is commonly seen in the aging immune system, known as 'inflamm-aging'. It is presently unclear how healthy macrophages are maintained throughout life and what connects inflammation with myeloid dysfunction during aging. Autophagy, an intracellular degradation mechanism, has known links with aging and lifespan extension. Here, we show for the first time that autophagy regulates the acquisition of major aging features in macrophages. In the absence of the essential autophagy gene Atg7, macrophage populations are increased and key functions such as phagocytosis and nitrite burst are reduced, while the inflammatory cytokine response is significantly increased - a phenotype also observed in aged macrophages. Furthermore, reduced autophagy decreases surface antigen expression and skews
\end{abstract}

\begin{tabular}{|c|c|}
\hline KARGER 125 & $\begin{array}{l}\text { (c) } 2015 \text { S. Karger AG, Basel } \\
1662-811 X / 15 / 0074-0375 \$ 0.00 / 0\end{array}$ \\
\hline $\begin{array}{l}\text { E-Mail karger@karger.com } \\
\text { www.karger.com/jin }\end{array}$ & $\begin{array}{l}\text { This is an Open Access article licensed under the terms of } \\
\text { the Creative Commons Attribution } 3.0 \text { Unported license } \\
\text { (CC BY 3.0) (www.karger.com/OA-license-WT), appli- } \\
\text { cable to the online version of the article only. }\end{array}$ \\
\hline
\end{tabular}

macrophage metabolism toward glycolysis. We show that macrophages from aged mice exhibit significantly reduced autophagic flux compared to young mice. These data demonstrate that autophagy plays a critical role in the maintenance of macrophage homeostasis and function, regulating inflammation and metabolism and thereby preventing immunosenescence. Thus, autophagy modulation may prevent excess inflammation and preserve macrophage function during aging, improving immune responses and reducing the morbidity and mortality associated with inflamm-aging.

(c) 2015 S. Karger AG, Basel

\section{Introduction}

Macroautophagy ('autophagy') is a vital intracellular degradation mechanism that regulates cellular and organismal homeostasis. Complete or tissue-specific ablation of autophagy genes in model organisms, including Cae-

\section{A.L.H. and I.P. contributed equally to this work.}

Dr. Anna Katharina Simon

MRC Human Immunology Unit, Weatherall Institute of Molecular Medicine University of Oxford

Oxford OX3 9DS (UK)

E-Mail katja.simon@ndm.ox.ac.uk 
norhabditis elegans, mice and non-human primates, have shown a consistent link between loss of autophagy, accelerated aging and reduced lifespan [1], whereas overexpression of the autophagy gene Atg 5 has been shown to increase lifespan [2]. Furthermore, several studies have implicated autophagy in protection against age-related diseases, such as Alzheimer's and Parkinson's disease [3]. The mechanisms by which autophagy is thought to increase lifespan and reduce age-related degeneration include removal of accumulated toxic protein aggregates, degradation of damaged mitochondria, and reduced cell death arising from enhanced homeostatic maintenance and hormesis [1]. Furthermore, autophagy limits stem cell damage and loss [4], and reduces oncogenic transformation, thereby preventing malignancy [5]. We have previously demonstrated that human $\mathrm{CD} 8^{+} \mathrm{T}$ cells show reduced autophagic flux with increasing donor age [6], indicating a role for autophagy in the aging adaptive immune system. However, few studies have investigated the role of autophagy in age-related changes in the innate immune system.

The immune system exhibits a significant decline in effective responses and a concordant increase in susceptibility to infection and attendant mortality with increasing age. Aging of the innate immune system is frequently accompanied by increased circulating neutrophils and macrophages [7, 8], and a general skewing of haematopoiesis toward the myeloid lineages, with the elderly significantly more susceptible to myeloproliferative and myelodysplastic syndromes than young individuals. Although many functions of innate immune cells decline with age, a chronic, low level of inflammation is observed in the elderly, which is a major contributor to age-associated frailty and morbidity, as well as increased mortality. This is caused by increased basal inflammatory cytokine production by innate cells such as macrophages, a process known as inflamm-aging [9].

Aging macrophages exhibit a variety of age-related functional and phenotypic changes, including a decrease in oxidative and nitric oxide (NO) burst and reduced phagocytic capacity [10]. Toll-like receptor (TLR) expression on macrophages decreases during aging [11], as does expression of antigen presentation receptors such as MHC II and the coreceptor CD86 [12, 13], potentially altering antigen presentation capacity and TLR expression. Multiple studies have also shown increased inflammatory cytokine production by aged macrophages $[14,15]$.

We have previously demonstrated that deletion of the essential autophagy gene Atg7 in the hematopoietic system (Vav-Atg7 ${ }^{-/-}$) results in significantly decreased $\mathrm{T}$ and $\mathrm{B}$ lymphocytes and declining stem cell numbers and capac- ity, yet increased numbers of circulating and tissue-resident myeloid cells [4]. In this study, we show that macrophages accumulate in the spleen of young Vav-Atg $7^{-/-}$ mice, reminiscent of aged mice. We show that loss of autophagy results in a variety of functional changes also reminiscent of those seen in aged macrophages, including impaired maturation, decreased antigen presentation capacity and reduced innate responses, alongside increased production of inflammatory cytokines at basal levels, following immune stimulation and even after phagocytosis. Furthermore, we find significantly decreased autophagy levels in macrophages from aged mice compared with macrophages derived from young mice. We show that aged macrophages have most features in common with autophagy-deficient macrophages and link functional changes in aged macrophages observed in the literature with the altered functions observed in $\mathrm{Atg} 7^{-/-}$macrophages. Loss of autophagy in macrophages appears to dramatically accelerate the appearance of aged macrophage features, suggesting that the decreased autophagy observed in aged macrophages contributes to their functional decline and aberrant inflammatory cytokine production, and implicating autophagy in the regulation of inflamm-aging.

\section{Materials and Methods}

Mice

Mice were bred and housed in filter top cages under SPF conditions at Biomedical Services Oxford, and fed ad libitum. Atg $7^{\text {flox/flox }}$ mice were obtained from M. Komatsu and Vav-iCre mice were kindly gifted by D. Kioussis, London, UK. Mice were bred on a C57BL/6 background and all procedures were carried out in accordance with the Home Office Animals (Scientific Procedures) Act 1986. VavAtg7 mice were generated by crossing Atg $7^{\text {flox/flox }}$ with Vav-iCre ${ }^{+/-}$ mice. The wild type (WT) mice used in the experiments were $\operatorname{Atg} 7^{\text {flox/flox; }}$ cre-, Atg $7^{\text {flox/wt; }}$ cre+ or Atg $7^{\text {flox/wt}}$; cre--, littermates of the $\operatorname{Atg} 7^{\text {flox/flox; }}$ cre+ $\left(\operatorname{Vav}-\operatorname{Atg} 7^{-/-}\right)$conditional knockout mice.

\section{Primary Cells}

Macrophages were obtained from a single cell suspension harvested from hind limb bones of 6 - to 8 -week-old mice and cultured in RPMI 1640 (Invitrogen) supplemented with FCS (10\%), glutamine, penicillin, streptomycin and $20 \mathrm{ng} / \mathrm{ml} \mathrm{recombinant} \mathrm{murine}$ M-CSF (Peprotech), and fed $50 \%$ of the initial volume of media on the third day of culture. Bone marrow macrophages (BMMФ) were stimulated on day 7 as described in the relevant figure legends. Peritoneal macrophages were obtained by peritoneal lavage with $5 \mathrm{ml}$ of PBS/5\% FCS, and kept on ice until use. Macrophages were identified in all experiments as $\mathrm{F} 4 / 80^{+} \mathrm{CD} 11 \mathrm{~b}^{+}$cells.

\section{$q P C R$}

mRNA was purified from cells by Trizol extraction (Invitrogen). The comparative $\mathrm{Ct}\left(\Delta \Delta \mathrm{C}_{\mathrm{T}}\right)$ method was used to evaluate relative gene expression in macrophages from $\mathrm{WT}$ and $\mathrm{Atg} 7^{-1-}$, or 
young and old mice using validated TaqMan ${ }^{\circledR}$ primer probes (Applied Biosystems) and TaqMan Gene Expression Mix (Applied Biosystems) in 96-well MicroAmp ${ }^{\circledR}$ reaction plates. Reactions were completed in the standard mode of a 7500 Fast Real-Time PCR system (Applied Biosystems). Gene expression was relative to WT or young expression levels, normalised to GAPDH.

\section{Electron Microscopy}

$\mathrm{F} 4 / 80^{+} \mathrm{BMM} \Phi$ were stained with anti-PE magnetic beads (Miltenyi), enriched using a MACS column (Miltenyi), and fixed for electron microscopy.

\section{Western Blot}

ВММФ were stimulated overnight and cells were lysed in Laemmli buffer (Pierce/Thermo Fisher Scientific). Supernatants were precipitated with 1 volume of methanol and 0.25 volumes of chloroform and the protein pellets were resuspended in Laemmli buffer. The samples were then separated on $15 \%$ polyacrylamide gels and transferred to nitrocellulose membranes (GE Healthcare). IL- $1 \beta$ was detected with a goat anti-IL- $1 \beta$ antibody (AF- $401 ; R \& D$ systems) and caspase 1 was detected with rabbit anti-mouse caspase 1 (sc-514; Santa Cruz Biotechnology). Cell lysates were separated on $12 \%$ polyacrylamide gels and blotted as above.

\section{Flow Cytometry and Image Stream}

Macrophages were incubated in Fc block (eBioscience) to reduce non-specific binding and stained with rat anti-mouse F4/80, CD11b, MHC II, MHC I, CD47, CD48, TLR4, CD86, CD14, GM$\mathrm{CSF}, \mathrm{IFN} \gamma \mathrm{R} 1$ (all eBioscience), rat anti-mouse mannose receptor (Biolegend), or mouse anti-human Glut1 (R\&D; confirmed crossreactive with mouse) at $4^{\circ} \mathrm{C}$ for $15 \mathrm{~min}$. Intracellular lipids were stained with Nile red ( $1 \mu \mathrm{M}$; Invitrogen). Dead cells were excluded using a live/dead exclusion marker (Invitrogen). For intracellular staining, protein secretion was blocked for $3 \mathrm{~h}$ using brefeldin A (eBioscience) and surface molecules were stained as above, followed by fixation and permeabilisation using commercially available buffers (eBioscience). Antibodies against IL-6, GM-CSF (eBioscience) or TNF- $\alpha$ (BD Pharmingen) were diluted in permeabilisation buffer and incubated with the cells at room temperature. Autophagy levels were evaluated using LC3 (MBL) and LysoID (Enzo) as previously described [6], or CytoID (Enzo Life Sciences) at 1:4,000 for $30 \mathrm{~min}$ at $37^{\circ} \mathrm{C}$. MitoTracker and MitoSOX staining were performed as in [16]. Cells were analysed using a Dako Cyan and BD LSRII flow cytometer or ImageStream 100 (Amnis). Data were analysed using FlowJo 9.0 or IDEAS 4.0.735.

\section{Phagocytosis of Beads or Apoptotic Cells}

Phagocytosis was evaluated using fluorescently labelled latex beads (Invitrogen). Macrophages were incubated with $1 \mu$ l beads \pm lipopolysaccharide (LPS) for $3 \mathrm{~h}$, and washed with PBS to remove loose beads. Surface-associated bead fluorescence was quenched by the addition of $0.4 \%$ Trypan blue (Invitrogen) diluted in PBS prior to flow cytometry [17]. For apoptotic cell assay, WT thymocytes were CFSE labelled (Life Technologies) and treated for $16 \mathrm{~h}$ with dexamethasone (Sigma), following which $\sim 50 \%$ of the thymocytes were apoptotic, as determined by annexin V (Invitrogen) and propidium iodine staining (eBioscience) [18]. After counting, thymocytes were incubated with macrophages for $2 \mathrm{~h}$ at $37^{\circ} \mathrm{C}$ in a $1: 3$ ratio. After $2 \mathrm{~h}$ of phagocytosis, brefeldin $\mathrm{A}$ was added to the culture for an additional $3 \mathrm{~h}$. The macrophages were then harvested, fixed and stained for TNF- $\alpha$ as described above. Control cells were quenched with Trypan blue $(50 \mu \mathrm{l})$ to confirm successful phagocytosis.

\section{Griess Reaction}

Macrophage culture supernatant was added to sulfanilamide solution and incubated for $10 \mathrm{~min}$ at room temperature (Promega). NED solution was then added to each well, and incubated for a further $10 \mathrm{~min}$ before being read at $550 \mathrm{~nm}$ on a plate reader. The nitrite concentration was determined by the standard curve.

\section{ELISA}

A commercially available kit (eBioscience) was used to detect IL- $1 \beta$ in the supernatant of macrophage cultures. The plate was read at $450 \mathrm{~nm}$ with the $595-\mathrm{nm}$ wavelength subtracted and the results analysed using Excel.

\section{In vivo Antigen Presentation}

ВМMФ were generated from male mice, as described above. Male dendritic cells (DCs) were used as a positive control and were derived using GM-CSF and IL-4 (both at $20 \mathrm{ng} / \mathrm{ml}$ ) in the same manner as the macrophages. WT male macrophages, DCs or Atg $7^{-1-}$ macrophages $\left(1 \times 10^{6}\right.$ cells per mouse $)$ were harvested on day 7, washed in PBS and injected into female recipients via the tail vein. The response was then boosted via vaccinia-UTY injection 7 days later and the antigen response was analysed via tetramerspecific anti-UTY CD8 ${ }^{+}$T cells. CD19 ${ }^{-} \mathrm{CD}^{+}$(both eBioscience) UTY tetramer-positive cells were identified by flow cytometry.

\section{Glucose Assay}

Supernatant samples were harvested following overnight LPS stimulation and diluted 1:25 to bring their glucose concentration within the optimal testing range of $20-80 \mu \mathrm{g} / \mathrm{ml}$ (R10 has a starting glucose concentration of $2 \mathrm{mg} / \mathrm{ml}$ ), along with D-glucose standards (Sigma). o-Dianisidine reagent was added to the glucose oxidase/ peroxidase reagent, added to each sample, and incubated at $37^{\circ} \mathrm{C}$ for $30 \mathrm{~min}$. Sulphuric acid solution was added and the absorbance at $540 \mathrm{~nm}$ was recorded using a plate reader. Values were normalised to the cell number by analysis of the protein content of the cells in each culture sample well.

\section{Seahorse}

Peritoneal macrophages were obtained by peritoneal lavage from 4 untreated Vav-Atg7 $7^{-/-}$or WT mice grown in RPMI at $2 \times$ $10^{6} / \mathrm{ml}$ with $10 \mu \mathrm{g} / \mathrm{ml}$ of M-CSF for $48 \mathrm{~h}$. Adherent cells were harvested and plated overnight at $2 \times 10^{5}$ cells/well in XF 24 -well cell culture microplates. Cells were washed in low-buffered bicarbonate-free DMEM ( $\mathrm{pH} 7.4)$ in a $\mathrm{CO}_{2}$-free incubator $\left(37^{\circ} \mathrm{C}\right)$ before rates of glycolysis were determined in 10 replicates for each genotype by measuring lactic acid release (extracellular acidification rate; ECAR) using an XF24 XF analyser (Seahorse Bioscience, Billerica, Mass., USA).

\section{Statistics}

Statistical significance was determined using the Mann-Whitney test, calculated using GraphPad Prism software. Mean values \pm $\mathrm{SD}$ are presented in the figures. $\mathrm{p}$ values $<0.05$ were considered to be significant; ${ }^{*} \mathrm{p}<0.05,{ }^{* *} \mathrm{p}<0.01,{ }^{* * *} \mathrm{p}<0.0001$ (one-way analysis of variance in fig. 1e, $4 \mathrm{f}, 5 \mathrm{a}, \mathrm{b}$, and $6 \mathrm{e}, \mathrm{f}$ ). 


\section{Results}

\section{Atg7-Deficient Macrophages Can Be Derived} Normally from Bone Marrow in vitro

In the Vav-Atg7 model, the excision of the essential autophagy gene Atg7 is driven by the hematopoietic stem cell-specific promoter Vav [16]. When macrophages were derived from bone marrow of Vav-Atg7 $7^{-/-}$mice, the excision was found to be $>93 \%$ efficient at the transcript level (fig. 1a) and no Atg7 protein was detected by Western blot (fig. 1b). ВМMФ can be derived in equal numbers from WT and Atg $7^{-/-}$mice bone marrow (fig. 1c), and express similar levels of the pan-myeloid marker CD11b and murine macrophage marker F4/80. Relatively increased numbers of macrophages/monocytes were present in the blood, spleen and peritoneum in Vav-Atg7 $7^{-/}$mice (fig. 1d), which was consistent with our earlier study showing generally increased myeloid cells [4]. Absolute numbers of $\mathrm{F} 4 / 80^{+} \mathrm{CD} 11 \mathrm{~b}^{+}$macrophages were increased significantly in the spleen only, but not in the blood or peritoneum (fig. 1e). Loss of Atg7 expression successfully inhibits autophagy, with significantly reduced formation of LC3-associated autophagosomes (fig. 1f) visible in $\mathrm{Atg}^{-/-}$ $B M M \Phi$, even following autophagy induction with LPS or IFN $\gamma$. To determine whether a lack of autophagy resulted in alterations to normal macrophage shape or size, morphology was assessed by electron microscopy. Increased vesiculation was visible in $\mathrm{Atg} 7^{-/-}$macrophages (fig. 1g), possibly contributed to by a 2 -fold increase in lipid droplets in $\operatorname{Atg} 7^{-/-}$ВMMФ (fig. 1h). Together, these data show that macrophages differentiate normally in the absence of autophagy.

\section{Decreased Autophagy Reduces Macrophage}

\section{Maturation and Adaptive Immune Function}

In order to effectively respond to immune stimuli such as LPS or IFN $\gamma$, mature macrophages upregulate expression of surface antigens such as the antigen presentationassociated molecules MHC II and CD86. Whereas MHC II expression was detectable on Atg $7^{-/-}$BMMФ and generally increased following stimulation, it was significantly lower on both unstimulated and stimulated $\mathrm{Atg} 7^{-/-}$ $\mathrm{BMM} \Phi$, relative to $\mathrm{WT}$ expression (fig. 2a, b). Similarly, while WT and $\mathrm{Atg} 7^{-1-}$ macrophages both expressed CD86 in the resting state, and exhibited increased expression following stimulation with LPS or IFN $\gamma, \operatorname{Atg}^{-/-}$macrophages expressed a significantly lower level of CD86 (fig. 2c, d). Expression of other immune-response-related proteins, such as MHC I and CD80, and adhesion molecules, such as CD47 and CD48, were also found to be sim- ilarly decreased on $\operatorname{Atg} 7^{-/-}$BMM $\Phi$ (table 1). To determine whether the reduction in surface marker expression was Atg7 specific or more generally regulated by autophagy, WT BMM $\Phi$ were treated with the autophagy inhibitor wortmannin. Treated WT macrophages expressed lower levels of MHC II, similar to Atg7 $7^{-/-}$macrophages (fig. 2e). Expression of CD86, TLR4 and MHC I showed a similar reduction after treatment with wortmannin (not shown). Conversely, induction of autophagy using rapamycin positively modulated surface antigen expression (fig. 2f). Lastly, Atg $5^{-/-}$macrophages showed a similar downregulation of surface molecules to Atg $7^{-/-}$macrophages (not shown). Isotype control stainings were performed under all conditions. As $A \operatorname{tg} 7^{-1-}$ macrophages generally displayed slightly higher autofluorescence than WT macrophages, the observed effect of lower surface marker expression in $\mathrm{Atg} 7^{-/-}$macrophages is thus likely underestimated rather than overestimated (data not shown).

Antigen presentation by professional APCs, such as macrophages, connects the innate immune system to the adaptive response. We used a male-antigen into female in vivo model to investigate whether loss of autophagy in macrophages influences the activation and expansion of male-specific $\mathrm{T}$ cells in female mice. The expansion of MHC I-restricted CD8 ${ }^{+} \mathrm{T}$ cells in this system is dependent on MHC II-restricted CD4 T cells [19]. Female recipients of male WT BMM $\Phi$ registered double the number of UTY-specific $\mathrm{CD}^{+} \mathrm{T}$ cells identified by MHC-I UTY tetramers compared to animals that received male Atg7 ${ }^{-/-}$BMMФ, indicating that Atg7-deficient macrophages did not efficiently present UTY antigen (fig. $2 \mathrm{~g}$ ). This could be as a result of impaired antigen processing,

Fig. 1. Atg7-deficient macrophages can be derived normally from bone marrow in vitro. a Deletion of Atg7 in BMMФ was confirmed by quantitative RT-PCR analysis, normalised to GAPDH. b Atg7 protein is not detectable in $A \operatorname{tg} 7^{-1-}$ macrophages. Western blot of WT and Atg7 ${ }^{-/-}$macrophage protein extracts. Each lane represents pooled protein extracts from $2 \mathrm{WT}$ or $2 \mathrm{Atg} 7^{-1-}$ macrophage cultures. $\mathrm{c} \mathrm{Atg} 7^{-1-}$ BMMФ express comparable levels of F4/80 and $\mathrm{CD} 11 \mathrm{~b}$, and are derived in similar numbers. d Proportions of $\mathrm{F} 4 / 80^{+} \mathrm{CD} 11 \mathrm{~b}^{+}$macrophages in the blood, spleen and peritoneum as detected by flow cytometry. e Absolute counts of F4/80 ${ }^{+} \mathrm{CD} 11 \mathrm{~b}^{+}$ macrophages in the spleen (left panel), blood and peritoneum (right panel). f $\mathrm{LC}^{+}$autophagosomes by immune fluorescence (left images) and proportions of macrophages with LC3+ autophagosomes, 40 cells were counted per slide. g F4/80+ macrophages enriched by MACS for electron microscopy. $\mathrm{N}=$ Nucleus. $\mathbf{h}$ Nile red staining of lipid droplets in $\mathrm{F} 4 / 80^{+} \mathrm{CD} 11 \mathrm{~b}^{+} \mathrm{BMM} \Phi$ as assessed by flow cytometry. FACS plots are representative of $>3$ experiments. Scale bar $=2 \mu \mathrm{m}$.

(For figure see next page.) 

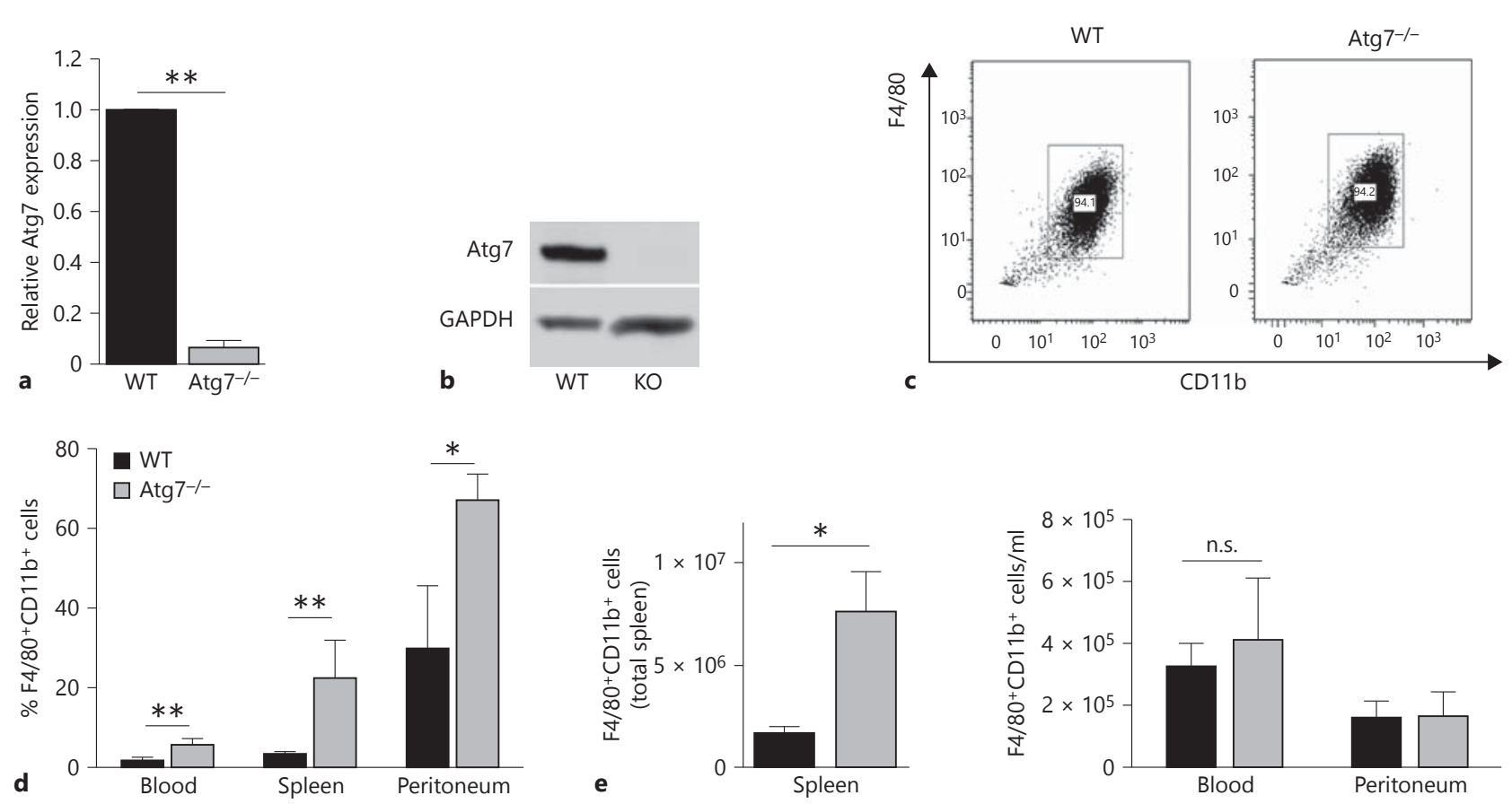

d
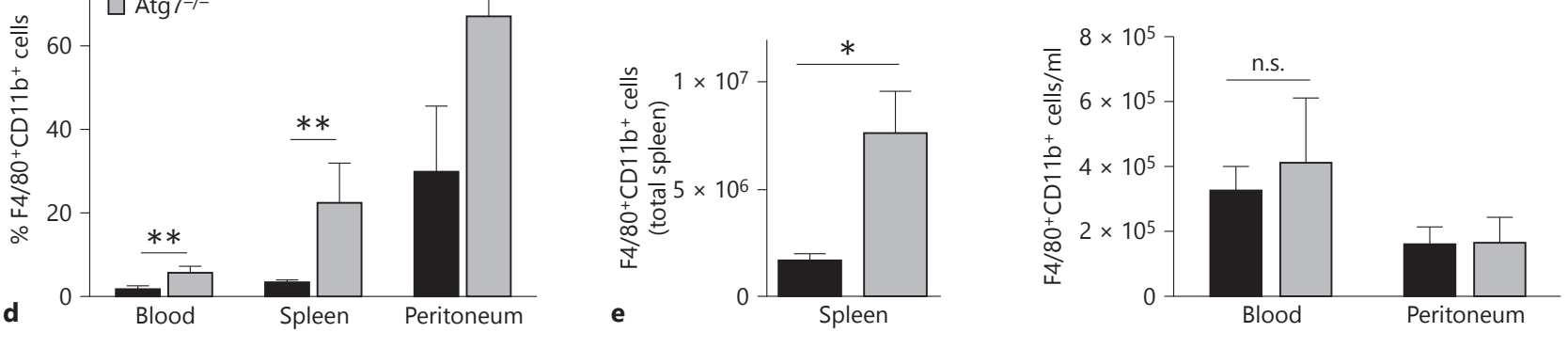

Unstimulated

LPS

IFNy
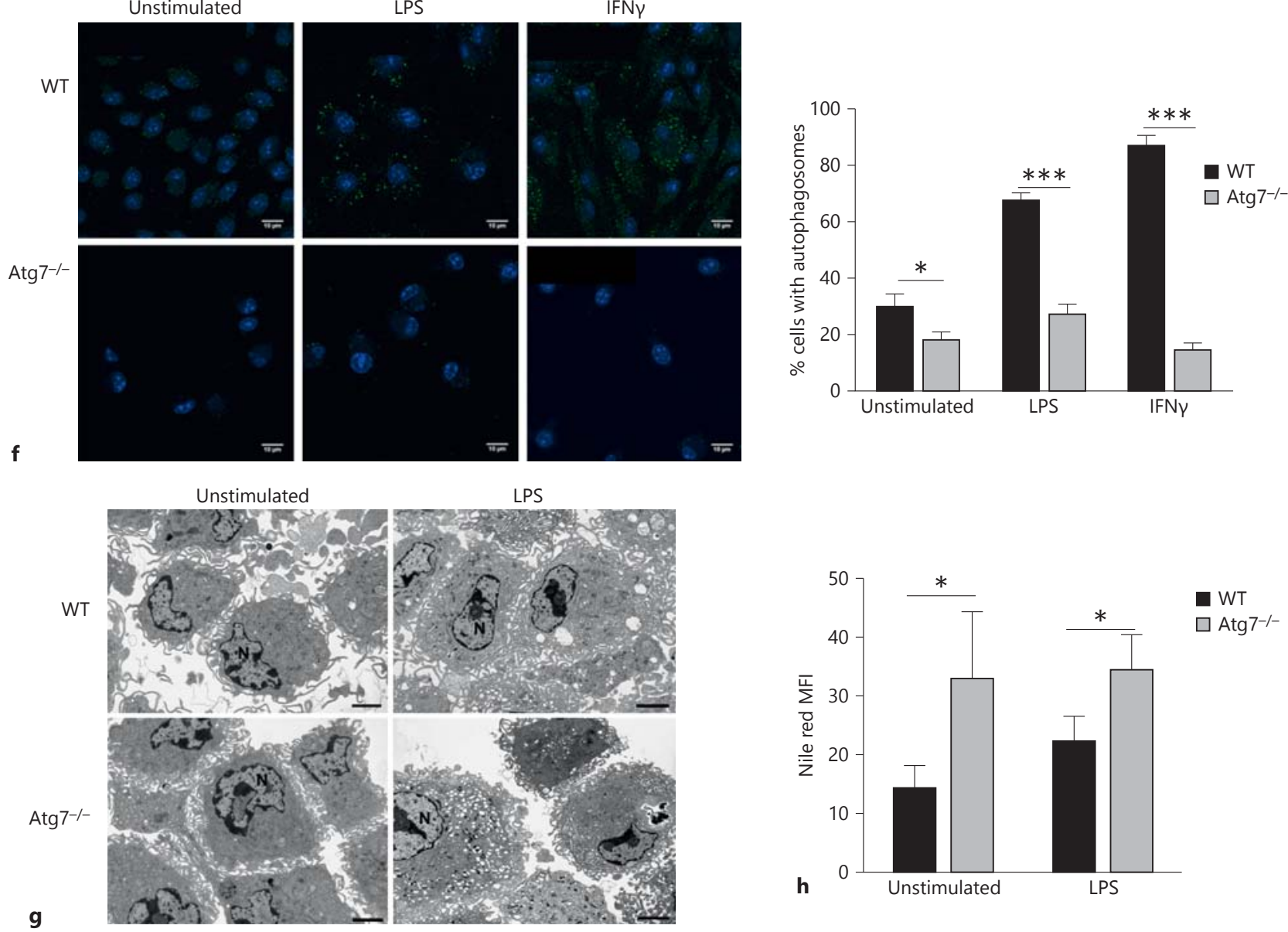

h

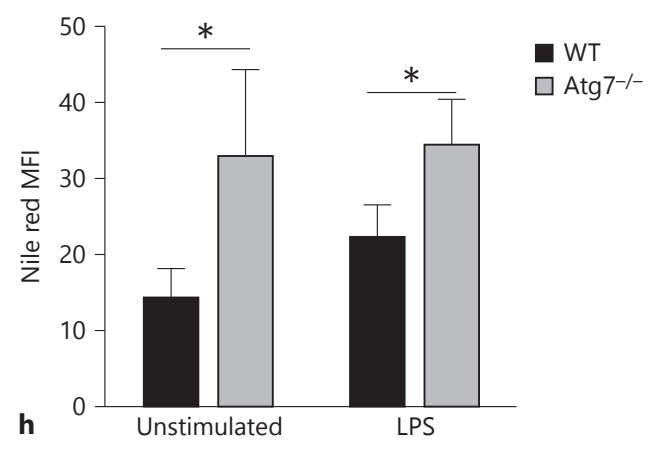



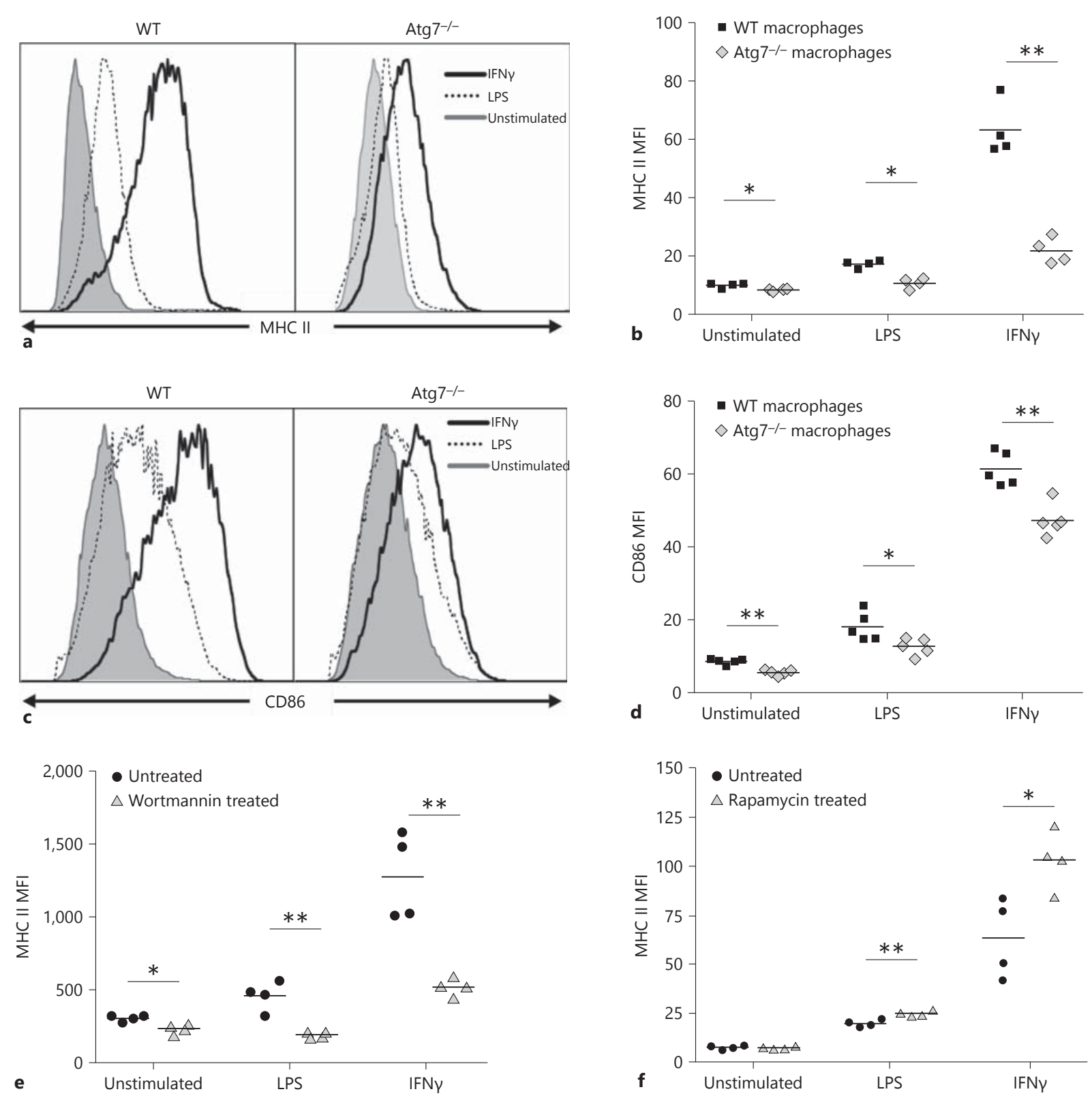

2
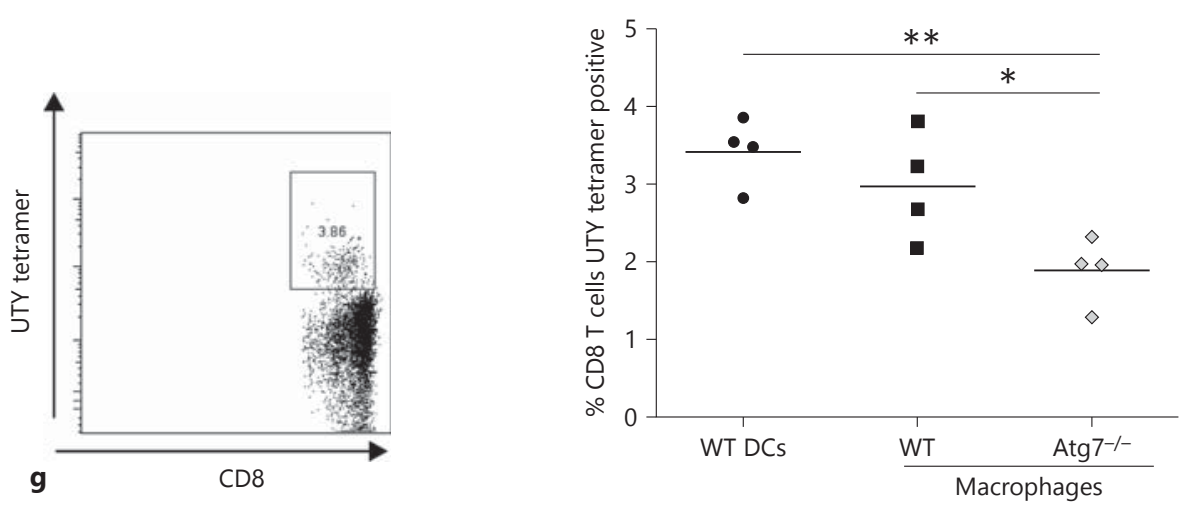

(For legend see next page.) 
which has been shown to involve autophagy [20], or as a result of reduced surface expression of MHC and costimulatory molecules.

\section{Decreased Autophagy Results in Reduced Innate Immune Function in Macrophages}

TLR4 is a pattern recognition receptor that is highly expressed on macrophages and recognises the bacterial endotoxin LPS. Signal transduction through TLR4, and its coreceptor CD14, causes differential expression of surface molecules and expression of cytokines. Similar to other surface molecules, TLR4 expression was lower on average on $\operatorname{Atg}^{-/-} \mathrm{BMM} \Phi$ for all stimuli. Furthermore,

Table 1. Summary of surface marker expression on autophagydeficient macrophages relative to WT

\begin{tabular}{ll}
\hline Marker & Atg7 $7^{-/-}$ \\
\hline F4/80 & $\pm / \bullet$ \\
CD11b & $\bullet$ \\
MHC I & $\downarrow$ \\
MHC II & $\downarrow$ \\
CD47 & $\downarrow$ \\
CD48 & $\downarrow$ \\
TLR4 & $\downarrow$ \\
CD86 & $\downarrow$ \\
Mannose receptor & $\downarrow$ \\
CD13 & \pm \\
CD14 & $\downarrow$ \\
M-CSFR & $\downarrow$ \\
\hline
\end{tabular}

The expression of each marker [decreased $(\downarrow)$, similar $(\bullet)$ or variable $( \pm)$ ] was determined by the geometric mean of fluorescence relative to WT macrophages.

Fig. 2. Reduced surface marker expression and antigen presentation in $\operatorname{Atg} 7^{-1-}$ macrophages. BMM $\Phi$ were stimulated overnight with LPS $(1 \mu \mathrm{g} / \mathrm{ml})$ or IFN $\gamma(10 \mathrm{ng} / \mathrm{ml})$ and stained for surface marker expression. a Reduced expression and upregulation of MHC II on Atg $7^{-/-}$macrophages. b Significantly reduced MHC II expression on $\mathrm{Atg}^{-/-}$macrophages. c, d CD86 expression is significantly reduced on $\mathrm{Atg} 7^{-/-}$macrophages. WT macrophages cultured with wortmannin $(100 \mathrm{nM}$; e) or rapamycin $(1 \mu \mathrm{M} ; \mathbf{f})$ for 7 days, stimulated overnight and stained for the surface expression of MHC II. g Male WT and Atg7 $7^{-/-}$macrophages and WT DCs (positive control) were injected i.v. into female recipients and male antigen specific $\mathrm{CD} 8^{+} \mathrm{T}$ cells were detected in recipients' blood using flow cytometry 2 weeks after the injection. UTY tetramer-positive cells shown are $\mathrm{CD} 19^{-} \mathrm{CD} 8^{+}$cells from a lysed blood sample (left plot), antigen presentation capacity was measured by the percentage of UTY-specific CD8 ${ }^{+} \mathrm{T}$ cells present in the blood of female mice (right plot).

Autophagy Regulates Aging in

Macrophages
TLR4 was not upregulated on autophagy-deficient BMM $\Phi$ following LPS stimulation, which was in contrast to WT macrophages where expression increased by almost $50 \%$ (fig. 3a). This could partly account for the failure of $\operatorname{Atg} 7^{--}$BMMФ to mature in response to LPS.

Given that macrophages are able to recognize a variety of pathogen-associated molecular patterns via different TLRs, in addition to TLR4, we investigated whether the maturation defect is extended to signalling in response to other TLR ligands. Atg7 $7^{-/}$BMMФ were stimulated overnight with heat-killed Listeria monocytogenes (HKLM), a TLR2 ligand, or polyinosinic:polycytidylic acid (poly I:C), a synthetic double-stranded RNA ligand to TLR3. Stimulation via alternate TLR pathways did not overcome the surface expression and maturation defect, with levels of MHC II expression on poly I:C or HKLM-stimulated macrophages significantly lower than on WT macrophages (fig. 3b). The surface markers CD86, MHC I, CD47 and CD48 were also found to be significantly lower in $\mathrm{Atg} 7^{-/-}$ macrophages following poly I:C and HKLM stimulation (data not shown), suggesting that this defect is not specific to signalling via TLR4. Finally, different concentrations of LPS were used to determine whether increasing the stimulus signal was sufficient to fully mature the macrophages. No tested LPS concentration was sufficient to bring Atg $7^{-/-}$ MHC II expression levels in line with their WT counterparts (fig. 3c). These data suggest that the maturational defect is not limited to specific stimulation pathways and cannot be overcome by saturating amounts of stimulation.

Macrophage stimulation using bacterial or inflammation-derived signals such as LPS and IFN $\gamma$ give rise to so called 'classically activated' or M1 macrophages, characterised by the production of inflammatory cytokines and upregulation of antigen presentation machinery. However, macrophages can also be programmed to an antiinflammatory phenotype using Th2-type signals such as IL-4 and IL-13, giving rise to alternately activated (M2) macrophages characterised by increased expression of the mannose scavenger receptor [21]. In order to determine whether the surface marker expression and upregulation defect was limited to M1 macrophages, BMMФ were stimulated with IL-4 and IL-13. Mannose receptor expression was significantly lower on $\mathrm{Atg}^{-/-} \mathrm{M} 2$ macrophages following stimulation with LPS or IL-4/IL-13 (fig. 3d), indicating that reduced maturation is not related to a single macrophage phenotype.

To determine whether reduced autophagy influenced innate macrophage functions, phagocytic capacity and nitrite burst were analysed in $\operatorname{Atg}^{-/-}$and WT BMMФ. The ability of ВМM $\Phi$ to phagocytose fluorescently la- 


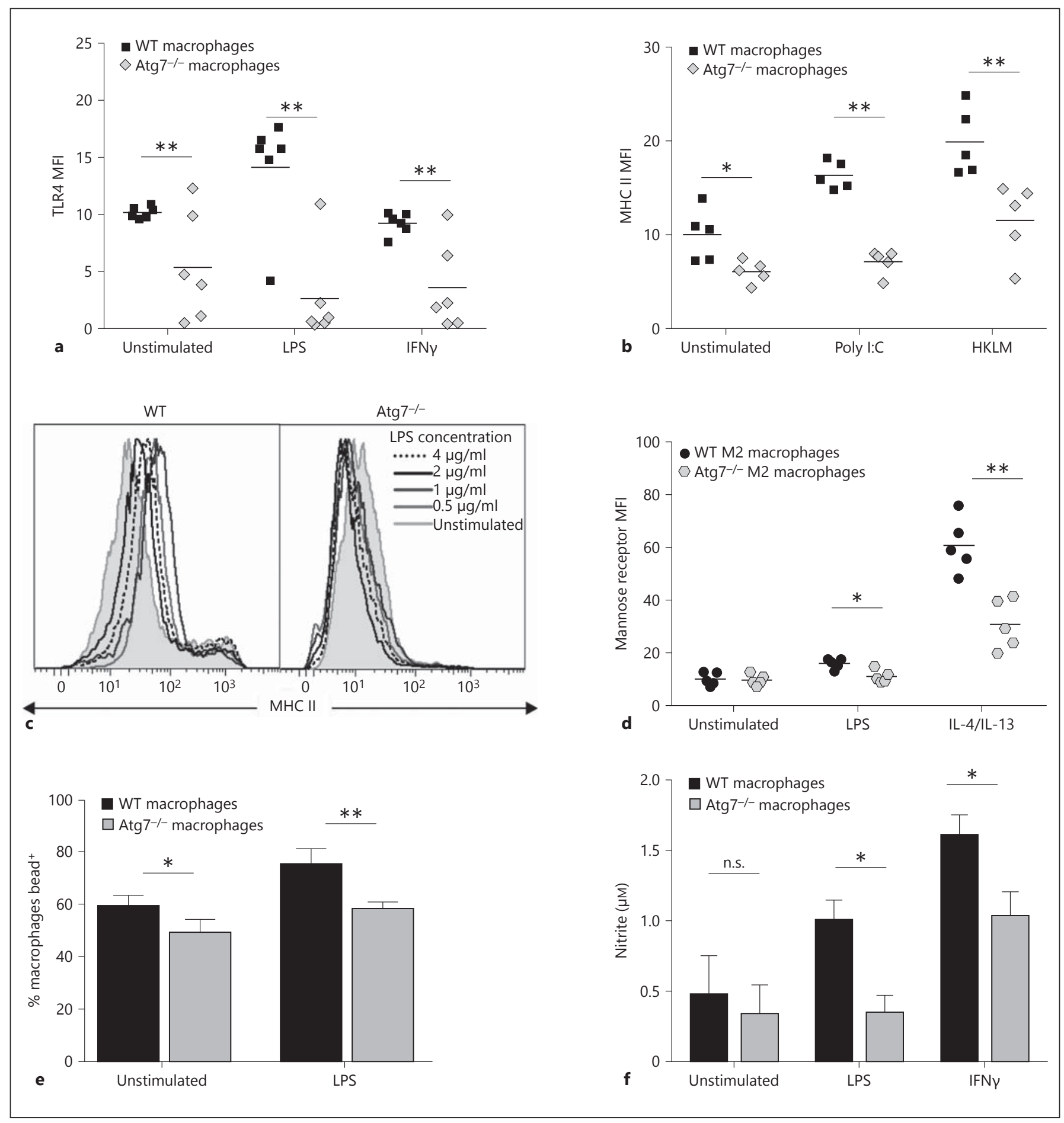

Fig. 3. Reduced innate immune function in $A \operatorname{tg} 7^{-/-}$macrophages. BMMФ were stimulated overnight with LPS $(0.5-4 \mu \mathrm{g} / \mathrm{ml})$, IFN $\gamma$ (10 ng/ml), poly I:C ( $1 \mu \mathrm{g} / \mathrm{ml})$, HKLM $\left(10^{8}\right.$ particles/well) or IL-4/ IL-13 (1 $\mu \mathrm{g}$ each/ml) prior to analysis by flow cytometry. a TLR4 expression on WT and Atg $7^{-/-}$macrophages. b MHC II expression on WT and $\mathrm{Atg}^{-1-}$ macrophages following stimulation of TLR3 and TLR2 with poly I:C and HKLM, respectively. c Representative histograms of MHC II expression following stimulation with in- creasing concentrations of LPS. d Mannose receptor expression following M2 stimulation using IL-4 and IL-13. e Phagocytic capacity was assessed by detection of green fluorescent beads taken up by macrophages using flow cytometry. The percentage of macrophages positive for internally located green fluorescence $3 \mathrm{~h}$ after the addition of beads to macrophage culture is depicted. $\mathbf{f}$ Nitrite concentration was analysed as an indicator of NO production using the Griess reaction. 
belled latex beads was assessed by flow cytometry. Unstimulated and LPS-stimulated Atg $7^{-/-}$macrophages exhibited a small but significant reduction in phagocytosis compared with their WT counterparts (fluorescence intensity relates to ingested beads as surface-associated bead fluorescence was quenched by Trypan blue; fig. 3e). Conversely, there was no difference observed between the ability of $A \operatorname{tg} 7^{-/-}$and WT macrophages in the uptake of CFSE-labelled apoptotic thymoyctes (online suppl. fig. 1; for all online suppl. material, see www.karger.com/ doi/10.1159/000370112).

$\mathrm{NO}$ is a short-lived free radical vital for the cytotoxic and microbicidal activity of macrophages. $B M M \Phi$ were stimulated overnight with LPS or IFN $\gamma$ and supernatants were analysed using the Griess reagent to identify secreted nitrite. Atg $7^{-/-}$and WT macrophages produced similar basal levels of NO. However, WT macrophages showed a significant increase in nitrite expression following LPS and IFN $\gamma$ stimulation, whereas Atg $7^{-/-}$macrophages showed no increase in NO production following LPS or only a small increase following IFN $\gamma$ (fig. 3f). A reduced nitrite burst following pathogenic stimuli coupled with some decreased phagocytic capacity could contribute to the reduced ability of autophagy-deficient macrophages to respond to and eliminate invading pathogens, thereby severely impeding the innate immune response to infection, similar to the phenotype observed in aged macrophages.

\section{Autophagy-Deficient Macrophages Exhibit High}

Levels of Basal Inflammation

As TLR4 expression was shown to be reduced in Atg7 ${ }^{-/-}$ macrophages, downstream signalling was assessed by investigating the expression of several cytokines following LPS stimulation using ELISA and intracellular flow cytometry. Atg $7^{-/-}$macrophages demonstrated increased secreted levels of IL- $1 \beta$ and inflammasome activation following LPS stimulation (fig. $4 \mathrm{a}, \mathrm{b}$ ), which is consistent with previous findings [22]. Interestingly, $\mathrm{Atg} 7^{-/-}$macrophages that had not received exogenous stimulation also exhibited increased IL- $1 \beta$ secretion (fig. $4 a$ ), detectable by the sensitive ELISA assay (fig. 4a) but not by Western blot (fig. 4b). Furthermore, the expression of other inflammatory cytokines, namely IL-6 (fig. 4d) and TNF- $\alpha$ (fig. 4e, $\mathrm{f}$, was also significantly increased in $\operatorname{Atg} 7^{-/-}$BMM $\Phi$, both at basal levels and following stimulation with LPS. Alternate activation also resulted in higher TNF- $\alpha$ and IL-6 production in $\mathrm{Atg} 7^{-/-}$macrophages (data not shown). In addition, we assessed the production of GM-CSF, a crucial growth factor regulating myeloid growth and development. Unstimulated and LPS-stimulated Atg $7^{-/-}$

Autophagy Regulates Aging in

Macrophages
BMMФ produce significantly more GM-CSF than WT BMMФ (fig. 4c). As it is well documented that ingestion of apoptotic cells by LPS-stimulated macrophages results in a significant diminution of TNF- $\alpha$ secretion by macrophages, we measured TNF- $\alpha$ after phagocytosis of apoptotic thymocytes. As opposed to LPS-stimulated WT macrophages, which display expected diminution of TNF- $\alpha$ production, LPS-stimulated Atg $7^{-1-}$ macrophages only slightly reduce TNF-a production following phagocytosis (fig. 4g, h, with similar levels of phagocytosis of apoptotic thymocytes by WT and $A \operatorname{tg} 7^{-/-}$; macrophages fig. 1a). Taken together these data show that although TLR4 and other signalling receptor expression is reduced in $A \operatorname{tg} 7^{-/-}$macrophages, signalling downstream of TLRs is intact and even amplified. These data emphasise the important role of autophagy in the regulation of inflammatory cytokine production and, even more crucially, indicate an indispensable requirement for autophagy in the prevention of unsolicited basal inflammation. This immature yet pro-inflammatory phenotype is reminiscent of immunosenescent macrophages.

\section{Loss of Autophagy Results in Altered Mitochondrial Function and Metabolism}

Accumulation of damage to DNA, protein and lipids caused by reactive oxygen species (ROS) is thought to be the dominant contributor to aging in post-mitotic cells, such as macrophages, caused by dysfunctional mitochondria leaking electrons from the respiratory chain [23]. As faulty mitochondria are degraded by autophagy [16], we sought to determine whether mitochondrial load and mitochondrial ROS (mROS) were altered in Atg $7^{-/-}$macrophages. Mitochondrial content was assessed using MitoTracker Green, which localises to mitochondria regardless of their membrane potential. Atg $7^{-/-}$macrophages had significantly higher mitochondrial content in both unstimulated and LPS-stimulated macrophages (fig. 5a). Generation of mROS was assessed using MitoSOX, a mitochondrial-targeting dye that is oxidised by mitochondrial superoxide producing fluorescence. Levels of mROS were significantly higher in Atg $7^{-/-}$macrophages than in WT macrophages (fig. 5b). These measurements were repeated in young (6-8 weeks) and old macrophages ( $>100$ weeks), demonstrating that senescent macrophages display the same phenotype as Atg $7^{-/-}$BMMФ with higher mitochondrial load and increased mROS production, but only following LPS stimulation (fig. 5c, d).

Under normal conditions, classically activated macrophages have relatively few mitochondria as they rely 

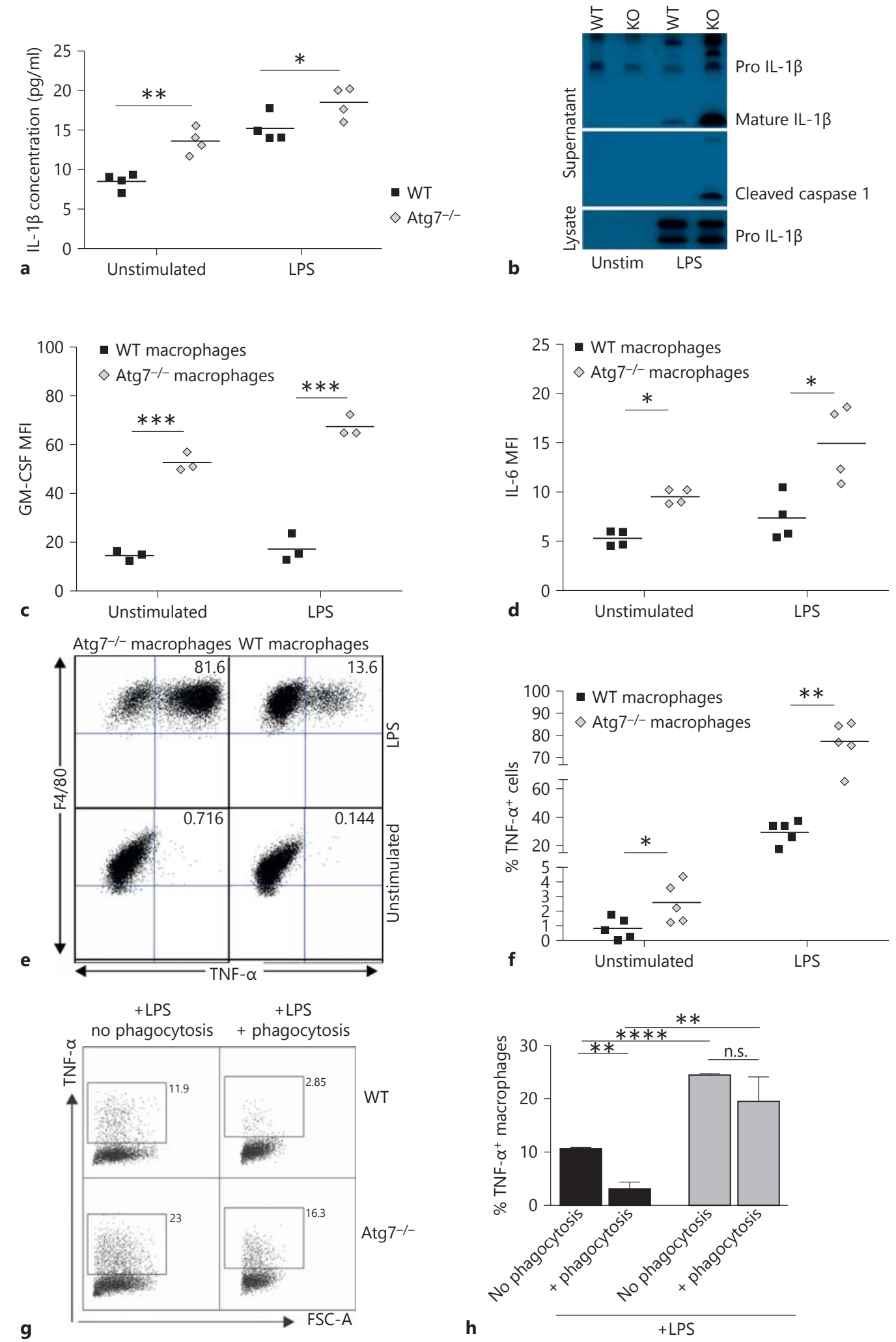

4

(For legend see next page.) 
primarily on glycolysis for ATP generation. In order to determine whether these mitochondrial changes influence macrophage metabolism, glycolysis levels were evaluated in $\mathrm{Atg} 7^{-1-}$ and WT macrophages using several complementary techniques. Glucose uptake by macrophages was assessed by assaying the concentration of glucose remaining in the medium following culture, relative to the glucose concentration in fresh medium $(2,000 \mu \mathrm{g} / \mathrm{l})$. Atg $7^{-/-}$macrophages utilise more glucose than WT macrophages, particularly following LPS stimulation (fig. 5e). Glucose is transported across the plasma membrane by the glucose transporter Glut-1, with increased expression required for increased glucose uptake. WT and Atg $7^{-/-}$macrophages both showed high levels of Glut-1 expression, reflecting the high energetic demands of macrophages. However, Atg $7^{-/-}$macrophages showed significantly higher expression of Glut-1 compared with WT macrophages, enabling their enhanced glucose uptake (fig. 5f). The glycolytic rate can also be assessed by observing changes in the ECAR, due to accumulation of lactic acid, a by-product of glycolysis. Analysis of supernatants using a Seahorse Bioscience XF24 analyser indicated a significantly higher basal ECAR (and hence glycolytic rate) in $A \operatorname{tg} 7^{-1}$ peritoneal macrophages, more than twice that of WT macrophages (fig. 5g). Hypoxia inducible-factors (HIF) are transcription factors that respond to decreased oxygen and co-ordinate adaptive responses to hypoxic environments, suppressing mitochondrial respiration that requires oxygen and enabling a switch to glycolytic production of ATP. HIF-1 a mRNA was similar in WT and $\mathrm{Atg}^{-/-}$macrophages but increased in LPS-stimulated Atg $7^{-1-}$ macrophages, suggesting that alterations in macrophage metabolism following loss of autophagy are initiated by activation of HIF-1a (fig. 5h), at least upon LPS stimulation.

\section{Senescent Macrophages Display Reduced Autophagic \\ Flux}

We next examined macrophages from aged $(>100$ weeks) C57Bl/6 mice. Similar to Vav-Atg7 ${ }^{-/-}$mice, mature $\mathrm{F} 4 / 80^{+}$macrophages were found to be relatively ac-

Fig. 4. Autophagy-deficient macrophages exhibit high levels of basal inflammation. a ELISA analysis of IL- $1 \beta$ in the supernatant of cultured BMМФ. b Inflammasome activation in LPS-stimulated macrophages, demonstrated by Western blot analysis of macrophage lysate (loading control) and supernatant. The blot has been cropped for clarity, with all visible bands retained. GM-CSF (c) and IL-6 (d) expression in LPS-stimulated WT and Atg $7^{-/-}$mac-

Autophagy Regulates Aging in

Macrophages cumulated in the blood and spleen of aged mice; however, aged mice had fewer peritoneal macrophages (fig. 6a). As in $A \operatorname{tg} 7^{-/-}$BMMФ, lipid droplets were increased in ВММФ from old mice (online suppl. fig. 2a). Furthermore, we found increased granularity by forward and side scatter using flow cytometry in both senescent and $\mathrm{Atg} 7^{-/-}$macrophages (online suppl. fig. $2 \mathrm{a}-\mathrm{d}$ ), possibly reflecting the increased lipid droplet content. Another similarity between senescent and Atg $7^{-1-}$ macrophages was the reduced surface expression of TLR4 and IFN $\gamma \mathrm{R} 1$, although this was more consistently observed across all stimulation conditions in $A \operatorname{tg} 7^{-/-}$macrophages than in aged macrophages, in which the effect was only observed for certain stimulation conditions (online suppl. fig. 3a-d).

To determine if reduced autophagy can account for the pre-inflammatory immature phenotype of senescent macrophages, we determined autophagy levels in senescent ВММФ. We found significant reductions in the expression of two essential autophagy genes, Beclin 1 (Atg6) and Atg7, in aged BMMФ by qPCR (fig. 6b). We then used an ImageStream-based method to detect autophagy levels in $B M M \Phi$ from old mice [6]. ВMM $\Phi$ were identified by $\mathrm{F} 4 / 80^{+} \mathrm{CD} 11 \mathrm{~b}^{+}$gating while LC3 puncta were enumerated, and LysoID intensity and the colocalisation (bright detail similarity; BDS) of these two markers was quantified (fig. 6e-g, examples in fig. $6 c$, d). The best correlate for autophagic flux is the colocalisation of LC3 and lysosomal markers, giving an indication of fusion between autophagosomes and lysosomes [6]. As expected, lysosomal inhibitors increased the colocalisation of LC3 and LysoID (fig. 6e). Aged BMMФ showed reduced autophagic flux under all conditions. Also as expected, stimulation of autophagy with LPS $+\mathrm{IFN} \gamma$ significantly increased the number of LC3 puncta/macrophage (LPS + IFN $\gamma$ is the best stimulus of autophagy in primary macrophages in our experience). However, aged macrophages displayed significantly fewer LC3 puncta than young macrophages, even following autophagic induction (fig. 6f). Lysosomal content was little affected by the treatments, and interestingly it was higher in BMMФ

rophages assessed by intracellular cytokine staining. e Representative FACS plots of intracellular TNF- $\alpha$ staining. f TNF- $\alpha$ expression is increased in $\mathrm{Atg} 7^{-/-}$macrophages, both basally and following LPS stimulation. $\mathbf{g}$ TNF- $\alpha$ expression in unstimulated and LPS-stimulated macrophages following phagocytosis of apoptotic thymocytes, with quantification over $\mathrm{n}=3$ mice in the bar graph (h). 


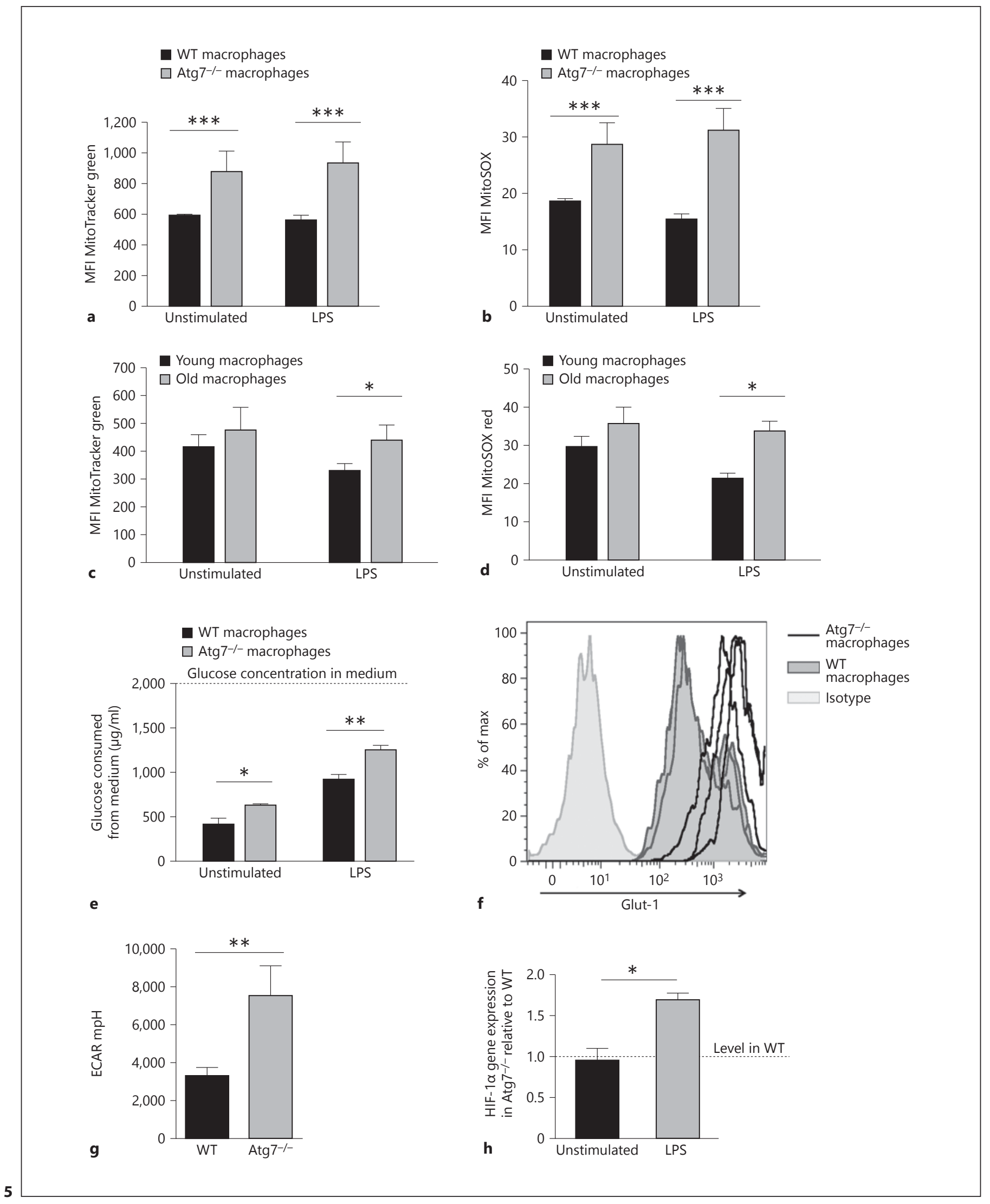

(For legend see next page.) 


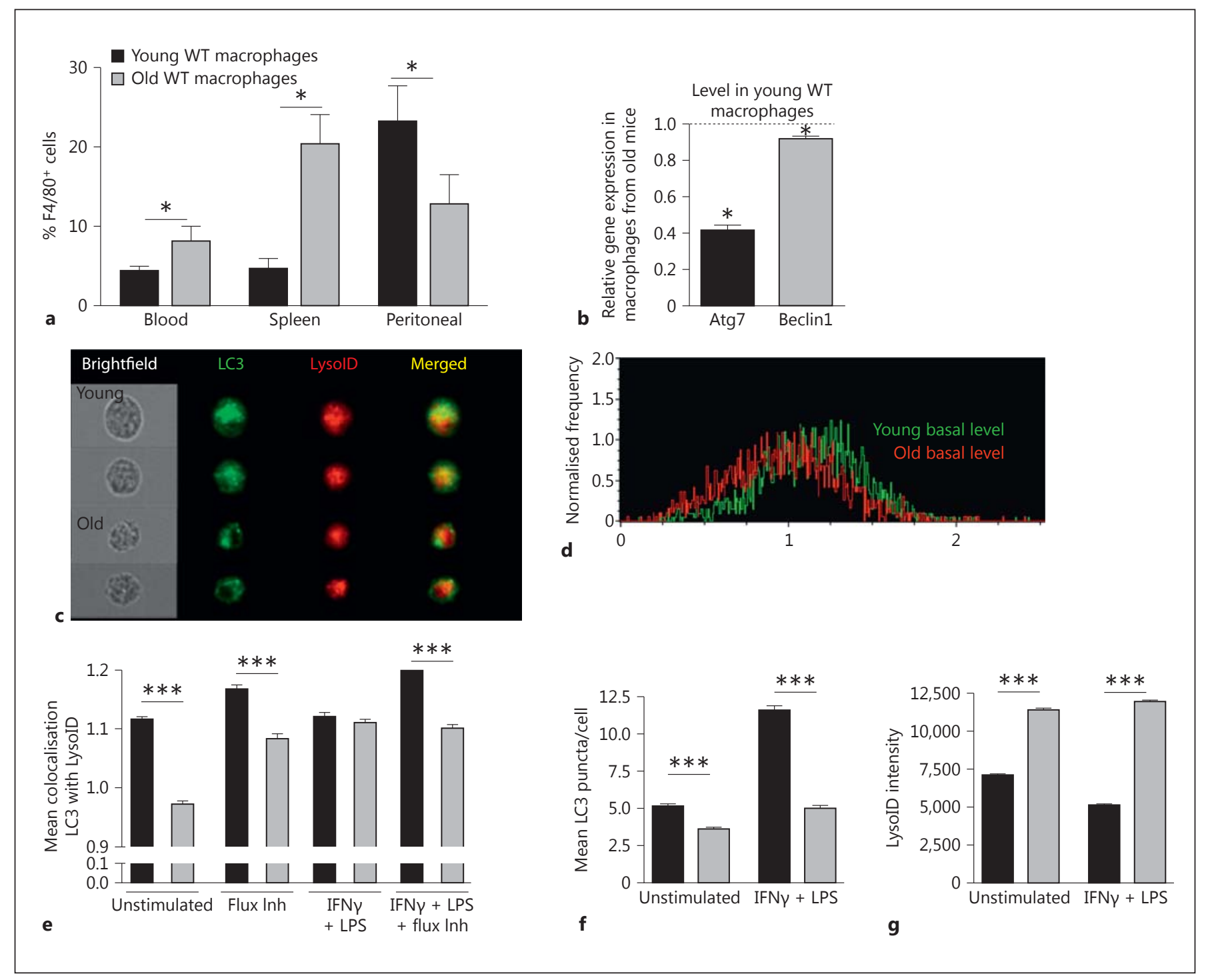

Fig. 6. Reduced autophagic flux in aged macrophages. a Frequency of $\mathrm{F} 4 / 80^{+} \mathrm{CD}_{11 \mathrm{~b}^{+}}$macrophages in blood, spleen and peritoneal suspensions from aged (107 weeks) and young ( 6 weeks) mice by flow cytometry. b Atg7 and Atg6 gene expression by qPCR analysis on RNA samples extracted from BMM $\Phi$ stimulated overnight with LPS $(1 \mu \mathrm{g} / \mathrm{ml})$. c-g BMM $\Phi$ were cultured for 7 days and stimulated overnight with IFN $\gamma(10 \mathrm{ng} / \mathrm{ml})$ and LPS $(1 \mu \mathrm{g} / \mathrm{ml})$ then stained for ImageStream, gating for single, live, F4/80-positive

Fig. 5. $\operatorname{Atg} 7^{-/-}$and senescent macrophages have an altered mitochondrial function and metabolism. a Mitochondrial burden in young (8 weeks) WT and $A \operatorname{tg} 7^{-/-}$macrophages assessed using MitoTracker Green dye and flow cytometric analysis. b Macrophage mitochondrial superoxide levels in WT and Atg $7^{-/-}$macrophages were analysed by staining with MitoSOX Red dye and flow cytometry. c Mitochondrial burden in aged macrophages (>100 weeks) as assessed by MitoTracker Green staining. d Mitochondrial superoxide levels in aged macrophages (>100 weeks) were assessed by MitoSOX red staining. e Atg $7^{-/-}$macrophages utilise cells. c Representative cell images showing brightfield, LC3, LysoID staining. d Representative histograms showing BDS (colocalisation) between LC3 and LysoID. e Mean BDS of LC3/LysoID, with or without IFN $\gamma /$ LPS treatment and/or autophagic flux inhibition as a positive control via 2 hour E64D $(10 \mu \mathrm{g} / \mathrm{ml})$ and pepstatin A $(2 \mu \mathrm{g} / \mathrm{ml})$ treatment. $\mathbf{f}$ Mean LC3 puncta per cell; spots were quantified via ImageStream. $\mathbf{g}$ LysoID mean intensity in macrophages following the indicated treatment.

higher levels of glucose than WT. The glucose concentration remaining in the media following culture was assessed and subtracted from the initial glucose concentration $(2,000 \mu \mathrm{g} / \mathrm{ml})$ to determine glucose consumption. $f$ Expression of the glucose receptor Glut-1 was assessed by flow cytometry. Histograms from 3 separate WT and $A \operatorname{tg} 7^{-/-}$macrophage cultures are shown overlaid. g Atg7 $7^{-/-}$peritoneal macrophages have higher levels of glycolysis as assessed by ECAR. $\mathbf{h}$ HIF-1a gene expression by qPCR analysis on RNA samples extracted from BMM $\Phi$ stimulated overnight with LPS $(1 \mu \mathrm{g} / \mathrm{ml})$. 
Table 2. Summary of similarities between aged and $\mathrm{Atg} 7^{-/-}$-deficient macrophages

\begin{tabular}{|c|c|c|}
\hline Aged macrophages & Macrophage phenotype & Atg $7^{-/-}$macrophages \\
\hline$\checkmark$ (not in peritoneum) & Relatively increased numbers of macrophages, monocytes [7] & $\checkmark$ \\
\hline$\checkmark$ (after LPS stimulation) & Increased mitochondria and mROS [45] & $\checkmark$ \\
\hline$\sqrt{ }$ & Decreased NO [32] & $\checkmark$ \\
\hline$\sqrt{ }$ & Reduced surface marker expression $[12,13]$ & $\checkmark$ \\
\hline$\checkmark$ & Reduced antigen presentation (DC) [46] & $\checkmark$ \\
\hline$\sqrt{ }$ & Metabolism alterations [41] & $\checkmark$ \\
\hline
\end{tabular}

Loss of autophagy in macrophages results in similar phenotypic changes to those previously described in aged macrophages.

from old compared to young mice in all conditions (fig. $6 \mathrm{~g}$ ), suggesting that changes in lysosomal content alone cannot explain reduced colocalisation. We also confirmed lower autophagy levels using CytoID cellular dye, an LC3-independent technique. CytoID labels autophagosomes and autolysosomes based on their relatively low $\mathrm{pH}$ (compared to the cytosol and other organelles), but spares lysosomes. We found that this dye was able to detect a small difference between LPS/IFN $\gamma$ stimulated young and old macrophages, whereas its sensitivity does not allow the difference to be detected in unstimulated cells (online suppl. fig. 4).

\section{Discussion}

Age-related immune senescence affects multiple cell types, resulting in increased cell death, decreased function and a lower capacity to prevent and clear infections. Loss of autophagy in macrophages causes multiple functional changes that are consistently associated with the development of senescence in aged macrophages. In this paper we have shown that the majority of the age-related changes in macrophages are mirrored in autophagy-deficient macrophages (summarised in table 2), and demonstrated that aged macrophages exhibit significantly reduced levels of autophagy, further underscoring the relationship between autophagy and aging and suggesting that loss of autophagy contributes to accelerated aging in macrophages. Dysfunctional aged macrophages are a major contributor to the aging process, and in particular to the low-grade pro-inflammatory phenotype that is associated with age-related diseases, e.g. atherosclerosis, obesity and type 2 diabetes.
We find that autophagy is dispensable for murine $\mathrm{F} 4 / 80^{+} \mathrm{CD} 11 \mathrm{~b}^{+}$macrophage differentiation, with no decrease in the number present in $\mathrm{Vav}-\mathrm{Atg} 7^{-/-}$mice in vivo, and with similar numbers derived from bone marrow culture in vitro. Indeed, $\mathrm{Vav}-\mathrm{Atg} 7^{-/-}$have a higher myeloid burden (including macrophages) than WT mice in the spleen, and a relatively higher myeloid burden in peritoneum and blood, suggesting that the absence of autophagy creates conditions that favour macrophage (and potentially other myeloid cell) differentiation and survival over lymphocytes. The spleen was also the organ in which we found the largest difference between numbers of macrophages in young and old mice. As aged hematopoietic stem cells (HSCs) are known to generate a myeloid-biased hematopoietic system [24], it is possible that loss of autophagy in HSCs, as present in this Vav-Atg7 mouse model, favours the generation of monocytes/macrophages over lymphocytes, thus contributing to increased myeloid cells in the spleen. However, HSCs (rather than the yolk sac progenitors) are the common ancestor to all three types of monocytes/macrophages analysed here (splenic, resident peritoneal and blood) [25], which partially argues against this. The alternative cell-intrinsic explanation would be that macrophages without autophagy proliferate more; indeed, new research suggests that tissue macrophages such as peritoneal and splenic macrophages self-renew and proliferate [25]. Thus, similar to other self-renewing cells such as HSCs, autophagy may help to keep macrophages quiescent [4].

Loss of Atg7 also appears not to significantly disrupt macrophage morphology. These findings are in contrast to two previous studies that identify a requirement for Atg7 during ex vivo macrophage development [26, 27]. However, the first study used the autophagy inhibitors 
3MA and chloroquine, which can cause autophagy-unrelated effects and toxicity, possibly explaining the different findings. Similarly, the second study used the enrichment of precursors prior to culture, a process that induces autophagy [6], potentially causing cell death in cells missing an intact autophagy pathway, thereby resulting in the reduction in $A \operatorname{tg} 7^{-/}$macrophages observed.

However, we have found that macrophage maturation and function is significantly impaired in the absence of autophagy. In addition to lower expression of vital surface markers such as MHC II, CD86 and TLR4 on unstimulated macrophages, we observed reduced upregulation of the same markers after maturational stimuli, regardless of the activation pathway targeted. The failure to efficiently upregulate these markers appears to be linked to changes in autophagy, rather than Atg7 specifically, and may represent a novel role for autophagy in maintaining or regulating functional endocytic pathways that control surface marker trafficking and expression. Aged macrophages also frequently exhibit a reduced expression of surface marker genes and receptors, including MHC II $[8,13]$ and TLR4 [11]. Upregulation of MHC I and II and coreceptors are crucial for efficient antigen presentation. Autophagy affects multiple aspects of antigen presentation by providing an intersection of exogenous or cytosolic antigen, via autophagosomes, with antigen-processing machinery in DCs. However, no research to date has specifically looked at the role of autophagy in macrophage antigen presentation. We used a male-antigen into female model to investigate whether loss of autophagy in macrophages influences MHC I-restricted antigen presentation to $\mathrm{CD}^{+} \mathrm{T}$ cells, which is known to rely on CD4 help [19]. Atg7 ${ }^{-/-}$macrophages did not efficiently present the male UTY antigen, confirming that autophagy is required for optimal antigen processing in macrophages as well as DCs, TECs and B cells. In addition to impaired antigen processing and delivery in the absence of autophagy $[20,28,29]$, reduced MHC and co-stimulatory molecule expression also likely plays a significant role in reduced antigen-specific responses, providing a second route whereby autophagy influences antigen-related immune responses.

The contribution of autophagy to phagocytosis remains ambiguous, with severely reduced phagocytosis by $A \operatorname{tg} 7^{-/-}$macrophages demonstrated under some circumstances [27], yet increased uptake in others [30]. In our hands, phagocytosis of beads, but not of apoptotic thymocytes, was reduced in the absence of autophagy, suggesting an indirect role for autophagy or Atg7 in this

Autophagy Regulates Aging in

Macrophages instance, possibly due to autophagy-related changes in phagocytic receptor expression or membrane motility [31]. Requirement of the latter may be different for the uptake of latex beads than for apoptotic thymocytes. We also demonstrate a significantly reduced nitrite burst in the absence of autophagy. Interestingly, macrophages from aged mice and elderly individuals have been shown to display a decline in $\mathrm{NO}$ production and phagocytosis in a variety of settings $[32,33]$. The age-related impairment is thought to be due to decreased scavenger and adhesion receptor expression and to changes in membrane motility and fluidity, both of which are also reflected in the reduced phagocytic capacity of autophagydeficient macrophages.

Autophagy has a well-established role in the modulation of inflammatory cytokine production through modulation of IL- $1 \beta$ processing and induction of the inflammasome [22]. However, in addition to increased inflammasome activation, we found significantly increased levels of TNF- $\alpha$, IL- 6, IL-12 and the inflammatory growth factor GM-CSF, suggesting a wider influence of autophagy in inflammasome-independent inflammatory cytokine and growth factor modulation. Notably, we found increased expression of such cytokines even in the absence of exogenous stimuli, suggesting an endogenous signal may be causing the observed inflammation. One candidate is mROS accumulation, which can induce proinflammatory cytokine expression [34]. Elevated levels of IL- 6 , TNF- $\alpha$ and IL- $1 \beta$ produced by inflammatory macrophages [14] are also frequently found in the serum and tissues of the elderly, resulting from inflammasome over-activation [35] and increased TLR signalling mediated by ROS and DAMPs released by stressed or dying cells [36]. Increased macrophage numbers are a common feature of aging in both mouse and human models [7, 8]. Accordingly, macrophages from old mice were significantly increased in the blood and spleen, similar to Vav-Atg7 mice. Chronic inflammatory and growth factor signalling in $\mathrm{Vav}-\mathrm{Atg} 7^{-/-}$macrophages may cause recruitment of myeloid cells into tissues and increase hematopoietic cell egress from the bone marrow, contributing to their increased numbers. It is clear that autophagy plays a vital role in the regulation and control of inflammatory cytokine production and, thus, it is likely that increased inflammatory signalling associated with aging is also exacerbated by age-associated reductions in autophagy and the corresponding increase in damaged mitochondria, ROS and cellular stress. Interestingly, our data suggest that even after phagocytosis, when TNF- $\alpha$ production usually subsides in WT macrophages in or- 
der to start scaling down the inflammatory response, Atg $7^{-/-}$macrophages continue to secrete TNF- $\alpha$ at higher levels. If falling autophagy levels with age result in continued TNF- $\alpha$ production rather than the expected postphagocytosis reduction, and phagocytosis of senescent cells becomes a major function of macrophages, then continued TNF- $\alpha$ secretion would exacerbate the lowgrade inflammation associated with inflamm-aging in a vicious circle.

In addition to an altered mitochondrial burden, we found that autophagy impacts on macrophage metabolism, increasing glucose uptake and the glycolytic rate. Activated M1 macrophages favour glycolysis for ATP generation [37], enabling prompt adaption to hypoxic inflammation sites, and thus generally high glycolysis is to be expected. However, the increased level of glycolysis in Atg $7^{-/-}$macrophages suggests that macrophages need increased levels of ATP or other glycolytic intermediates in the absence of autophagy, potentially to maintain mitochondria potential and avoid apoptosis, or to make up the energy/metabolite deficit, normally supplied by autophagy [38]. A higher mitochondrial load and ROS were only detected in LPS-stimulated senescent macrophages, and not in unstimulated macrophages, possibly because the differences are subtle when autophagy levels are deceased (senescent) rather than abolished $\left(\mathrm{Atg} 7^{-/-}\right)$, and are only detectable under stress such as with LPS. Furthermore, Glut-1 overexpression and increased glycolysis are known to result in a hyper-inflammatory state [39], suggesting a link between the inflammatory phenotype of Atg $7^{-/-}$ macrophages and their increased rate of glycolysis. ROS and inflammatory cytokines, both of which are features of Atg $7^{-/-}$and aged macrophages, are known to increase HIF-1 a expression [40], a transcriptional driver of glycolysis, potentially altering metabolism in aged macrophages as well. Indeed, chronic glycolysis has been hypothesised as a driver of aging [41]. On the other hand, it is an intriguing possibility that HIF-1a governs the in- creased inflammatory response (elevated IL-1 $\beta$, IL- 6 and TNF- $\alpha$ ) such as is shown for DCs [42].

Autophagy levels decline in many tissues with age [1, 6], causing increased ROS and oxidative stress. Accordingly, we found significantly reduced levels of autophagy in macrophages derived from aged mice and in the expression of key autophagy genes, suggesting that age-related changes in autophagy may be related to the accumulation of genetic changes over time. However, the precise mechanisms by which aging results in reduced autophagy remains unclear, with hypotheses including altered autophagic signalling and reduced autophagosome clearance by the lysosomal system [43]. Our data suggest that although lysosomal content is increased, the number of autophagosomes and autolysomes is reduced with age. It is known that dysfunctional lysosomes filled with nondegradable material such as lipofuscin accumulate with age [43]. Inducing autophagy in aged cells and organisms by caloric restriction or chemical modulation [44] reduces aging-related damage, suggesting that age-related decline in macrophage function may be prevented by maintaining autophagy levels throughout life, or potentially even partially rescued by the induction of autophagy in aged macrophages. Our study suggests that a mild upregulation of autophagy in the aging organism may help to arrest the decline in immune function and thereby prevent the development of chronic inflammation associated with aging.

\section{Acknowledgements}

The authors acknowledge the John Radcliffe Biomedical Services staff for their animal care, the flow cytometry unit at the Weatherall Institute of Molecular Medicine for sorting, and Prof. Marion MacFarlane and Prof. Kelvin Cain for their assistance with Seahorse and metabolic analysis. This work was funded by the Nuffield Department of Clinical Medicine, the Wellcome Trust and the MRC Human Immunology Unit, Oxford.

\section{References}

1 Rubinsztein DC, Marino G, Kroemer G: Autophagy and aging. Cell 2011;146:682-695.

$>2$ Pyo JO, Yoo SM, Ahn HH, Nah J, Hong SH, Kam TI, Jung S, Jung YK: Overexpression of Atg5 in mice activates autophagy and extends lifespan. Nat Commun 2013;4:2300.

$>3$ Nixon RA: The role of autophagy in neurodegenerative disease. Nat Med 2013;19:983997.
4 Mortensen M, Soilleux EJ, Djordjevic G, Tripp R, Lutteropp M, Sadighi-Akha E, Stranks AJ, Glanville J, Knight S, Jacobson SE, Kranc KR, Simon AK: The autophagy protein Atg7 is essential for hematopoietic stem cell maintenance. J Exp Med 2011;208:455-467.

$>5$ Degenhardt K, Mathew R, Beaudoin B, Bray K, Anderson D, Chen G, Mukherjee C, Shi Y, Gelinas C, Fan Y, Nelson DA, Jin S, White E: Autophagy promotes tumor cell survival and restricts necrosis, inflammation, and tumorigenesis. Cancer Cell 2006;10:51-64.
-6 Phadwal K, Alegre-Abarrategui J, Watson AS, Pike L, Anbalagan S, Hammond EM, WadeMartins R, McMichael A, Klenerman P, Simon AK: A novel method for autophagy detection in primary cells: impaired levels of macroautophagy in immunosenescent $\mathrm{T}$ cells. Autophagy 2012;8:677-689.

7 Boggs D, Patrene K, Steinberg H: Aging and hematopoiesis. VI. Neutrophilia and other leukocyte changes in aged mice. Exp Hematol 1986;14:372-379. 
8 Strohacker K, Breslin WL, Carpenter KC, McFarlin BK: Aged mice have increased inflammatory monocyte concentration and altered expression of cell-surface functional receptors. J Biosci 2012;37:55-62.

-9 Franceschi C, Capri M, Monti D, Giunta S, Olivieri F, Sevini F, Panourgia MP, Invidia L, Celani L, Scurti M, Cevenini E, Castellani GC, Salvioli S: Inflammaging and anti-inflammaging: a systemic perspective on aging and longevity emerged from studies in humans. Mech Ageing Dev 2007;128:92-105.

10 Koike E, Kobayashi T, Mochitate K, Muraka$\mathrm{mi}$ M: Effect of aging on nitric oxide production by rat alveolar macrophages. Exp Gerontol 1999;34:889-894.

11 Renshaw M, Rockwell J, Engleman C, Gewirtz A, Katz J, Sambhara S: Cutting edge: impaired toll-like receptor expression and function in aging. J Immunol 2002;169:4697-4701.

12 van Duin D, Mohanty S, Thomas V, Ginter S, Montgomery RR, Fikrig E, Allore HG, Medzhitov R, Shaw AC: Age-associated defect in human TLR-1/2 function. J Immunol 2007;178:970-975.

13 Herrero C, Marques L, Lloberas J, Celada A: IFN- $\gamma$-dependent transcription of MHC class II IA is impaired in macrophages from aged mice. J Clin Invest 2001;107:485-493.

14 Bruunsgaard H, Pedersen M, Pedersen BK: Aging and proinflammatory cytokines. Curr Opin Hematol 2001;8:131-136.

-15 Henry CJ, Huang Y, Wynne AM, Godbout JP: Peripheral lipopolysaccharide (LPS) challenge promotes microglial hyperactivity in aged mice that is associated with exaggerated induction of both pro-inflammatory IL- $1 \beta$ and anti-inflammatory IL-10 cytokines. Brain Behav Immun 2009;23:309-317.

16 Mortensen M, Ferguson DJ, Edelmann M, Kessler B, Morten KJ, Komatsu M, Simon AK: Loss of autophagy in erythroid cells leads to defective removal of mitochondria and severe anemia in vivo. Proc Natl Acad Sci U S A 2010;107:832-837.

17 Nuutila J, Lilius EM: Flow cytometric quantitative determination of ingestion by phagocytes needs the distinguishing of overlapping populations of binding and ingesting cells. Cytometry A 2005;65:93-102.

-18 Aziz M, Yang WL, Wang P: Measurement of phagocytic engulfment of apoptotic cells by macrophages using pHrodo succinimidyl ester. Curr Protoc Immunol 2013, unit 14.31.

19 Millrain M, Scott D, Addey C, Dewchand H, Ellis P, Ehrmann I, Mitchell M, Burgoyne P, Simpson E, Dyson J: Identification of the immunodominant $\mathrm{HY} \mathrm{H} 2-\mathrm{D}^{\mathrm{k}}$ epitope and evaluation of the role of direct and indirect antigen presentation in HY responses. J Immunol 2005;175:7209-7217.
20 Paludan C, Schmid D, Landhalter M, Vockerodt M, Kube D, Tuschl T, Munz C: Endogenous MHC class II processing of a viral nuclear antigen after autophagy. Science 2005; 307:593-596.

21 Gordon S: Alternative activation of macrophages. Nat Rev Immunol 2003;3:23-35.

22 Saitoh T, Fujita N, Jang MH, Uematsu S, Yang BG, Satoh T, Omori H, Noda T, Yamamoto N, Komatsu M, Tanaka K, Kawai T, Tsujimura T, Takeuchi O, Yoshimori T, Akira S: Loss of the autophagy protein Atg16L1 enhances endotoxin-induced IL- $1 \beta$ production. Nature 2008;456:264-268.

23 Murphy MP: How mitochondria produce reactive oxygen species. Biochem J 2009;417:1-13.

24 Sudo K, Ema H, Morita Y, Nakauchi H: Ageassociated characteristics of murine hematopoietic stem cells. J Exp Med 2000;192:12731280.

25 Sieweke MH, Allen JE: Beyond stem cells: selfrenewal of differentiated macrophages. Science 2013;342:1242974.

26 Zhang Y, Morgan MJ, Chen K, Choksi S, Liu $\mathrm{ZG}$ : Induction of autophagy is essential for monocyte-macrophage differentiation. Blood 2012;119:2895-2905.

27 Jacquel A, Obba S, Boyer L, Dufies M, Robert G, Gounon P, Lemichez E, Luciano F, Solary E, Auberger P: Autophagy is required for CSF-1-induced macrophagic differentiation and acquisition of phagocytic functions. Blood 2012;119:4527-4531.

-28 Dengjel J, Schoor O, Fischer R, Reich M, Kraus M, Muller M, Kreymborg K, Altenberend F, Brandenburg J, Kalbacher H, Brock R, Driessen C, Rammensee HG, Stevanovic S: Autophagy promotes MHC class II presentation of peptides from intracellular source proteins. Proc Natl Acad Sci U S A 2005;102: 7922-7927.

29 Lee HK, Mattei LM, Steinberg BE, Alberts P, Lee YH, Chervonsky A, Mizushima N, Grinstein $\mathrm{S}$, Iwasaki $\mathrm{A}$ : In vivo requirement for Atg5 in antigen presentation by dendritic cells. Immunity 2010;32:227-239.

- 30 Bonilla DL, Bhattacharya A, Sha Y, Xu Y, Xiang Q, Kan A, Jagannath C, Komatsu M, Eissa NT: Autophagy regulates phagocytosis by modulating the expression of scavenger receptors. Immunity 2013;39:537-547.

31 Kadandale P, Stender JD, Glass CK, Kiger AA: Conserved role for autophagy in Rhol-mediated cortical remodeling and blood cell recruitment. Proc Natl Acad Sci U S A 2010;107: 10502-10507.

32 Khare V, Sodhi A, Singh SM: Effect of aging on the tumoricidal functions of murine peritoneal macrophages. Nat Immun 1996;15:285-294.

33 Cannizzo ES, Clement CC, Sahu R, Follo C, Santambrogio L: Oxidative stress, inflammaging and immunosenescence. J Proteomics 2011;74:2313-2323.
34 Naik E, Dixit VM: Mitochondrial reactive oxygen species drive proinflammatory cytokine production. J Exp Med 2011;208:417-420.

35 Salminen A, Kaarniranta K, Kauppinen A: Inflammaging: disturbed interplay between autophagy and inflammasomes. Aging 2012;4: 166-175.

36 Gill R, Tsung A, Billiar T: Linking oxidative stress to inflammation: toll-like receptors. Free Radic Biol Med 2010;48:1121-1132.

37 Pearce EL, Pearce EJ: Metabolic pathways in immune cell activation and quiescence. Immunity 2013;38:633-643.

38 Lunt SY, Vander Heiden MG: Aerobic glycolysis: meeting the metabolic requirements of cell proliferation. Annu Rev Cell Dev Biol 2011;27:441-464.

- 39 Freemerman AJ, Johnson AR, Sacks GN, Milner JJ, Kirk EL, Troester MA, Macintyre AN, Goraksha-Hicks P, Rathmell JC, Makowski L: Metabolic reprogramming of macrophages: glucose transporter 1 (GLUT1)-mediated glucose metabolism drives a proinflammatory phenotype. J Biol Chem 2014;289:7884-7896.

40 Gerald D, Berra E, Frapart YM, Chan DA, Giaccia AJ, Mansuy D, Pouyssegur J, Yaniv M, Mechta-Grigoriou F: JunD reduces tumor angiogenesis by protecting cells from oxidative stress. Cell 2004;118:781-794.

41 Hipkiss AR: Does chronic glycolysis accelerate aging? Could this explain how dietary restriction works? Ann NY Acad Sci 2006;1067: 361-368.

42 Everts B, Amiel E, Huang SC, Smith AM, Chang CH, Lam WY, Redmann V, Freitas TC, Blagih J, van der Windt GJ, Artyomov MN, Jones RG, Pearce EL, Pearce EJ: TLRdriven early glycolytic reprogramming via the kinases TBK1-IKKe supports the anabolic demands of dendritic cell activation. Nat Immunol 2014;15:323-332.

43 Cuervo AM, Bergamini E, Brunk UT, Droge W, Ffrench M, Terman A: Autophagy and aging: the importance of maintaining 'clean' cells. Autophagy 2005;1:131-140.

-44 Trepanowski JF, Canale RE, Marshall KE, Kabir MM, Bloomer RJ: Impact of caloric and dietary restriction regimens on markers of health and longevity in humans and animals: a summary of available findings. Nutr J 2011;10:107.

45 Salminen A, Ojala J, Kaarniranta K, Kauppinen A: Mitochondrial dysfunction and oxidative stress activate inflammasomes: impact on the aging process and age-related diseases. Cell Mol Life Sci 2012;69:2999-3013.

46 Wong CP, Magnusson KR, Ho E: Increased inflammatory response in aged mice is associated with age-related zinc deficiency and zinc transporter dysregulation. J Nutr Biochem 2013;24:353-359. 